\title{
Endoparásitos de micromamíferos del noroeste de Perú. 1: helmintos de marsupiales
}

\author{
Endoparasites of small mammals from northeastern Peru. 1: Helmintes of \\ marsupials
}

Manuel Tantaleán ${ }^{1}, M_{0}$ ica Díaz ${ }^{2 *}$, Nofre Sánchez ${ }^{3}$ y Harold Portocarrero ${ }^{4}$

\begin{abstract}
${ }^{*}$ Corresponding author
1 Laboratorio de Parasitología, Facultad de Medicina Veterinaria y Zootecnia, Universidad Peruana Cayetano Heredia. Lima, Perú. E-mail: mtantaleanv@hotmail.com 2 Conicet (Consejo de Investigaciones Científicas y Técnica) and Pidba (Programa de Investigaciones de Biodiversidad Argentina), Universidad Nacional de Tucumán, Argentina, Miguel Lillo 255, 4000, Tucumán, Argentina. E-mail: mmonicadiaz@arnet.com.ar

3 IVITA-Iquitos, Facultad de Medicina Veterinaria, Universidad Nacional Mayor de San Marcos. E-mail: nofresp@hotmail.com

4 Fundación Iquitos Centro de Rescate Amazónico ACOBIADWAZOO (Asociación para la Conservación de la Biodiversidad Amazónica) Jr. Galves \#1506. Instituto de investigaciones de la Amazonia Peruana (IIAP). Carretera Iquitos Nauta km 4.5. E-mail: haroldpz10@yahoo.es
\end{abstract}

Presentado: $\quad 04 / 12 / 2009$ Aceptado: $\quad 14 / 05 / 2010$ Publicado online: 14/12/2010

\section{Resumen}

En este trabajo, informamos los resultados del análisis parasitológico realizado a 40 individuos marsupiales de las especies Caluromys lanatus, Didelphis marsupialis, Marmosops noctivagus, Metachirus nudicaudatus, Marmosa (Micoureus) regina, Monodelphis adusta, Philander andersoni y Philander opossum procedentes del departamento de Loreto, Perú. Se determinaron en total 11 especies de helmintos parásitos: Nematoda: Aspidodera sp., Cruzia tentaculata, Physaloptera mirandai, Physaloptera sp., Pterygodermatites sp., Trichuris sp., Turgida turgida, y Viannaia sp.; Trematoda: Podospathalium pedatum; Acanthocephala: Giganthorhynchus ortizi; y Pentastomida: ninfa. Los parásitos Trichuris sp., Pterygodematities sp., Turgida turgida, Viannaia sp. y Podospathalium pedatum son nuevos registros para el Perú. De igual manera, se registran por primera vez las siguientes asociaciones parásitos-huéspedes: Pterygodermatites sp.-Marmosa regina, Viannaia sp.Marmosops noctivagus, Trichuris sp.-Marmosops cf. noctivagus, Podospathalium pedatum-Monodelphis adusta, Giganthorhynchus ortizi-Marmosops cf. noctivagus, y ninfas de pentastómidos-Marmosa regina y Metachirus nudicaudatus.

Palabras clave: vida silvestre, parásitos, marsupiales, Amazonia, Loreto, Perú.

\section{Abstract}

In this paper, we report the results of parasitological examination performed on 40 specimens of marsupials species: Caluromys lanatus, Didelphis marsupialis, Marmosops noctivagus, Metachirus nudicaudatus, Marmosa (Micoureus)regina, Monodelphis adusta, Philander andersoni and Philander opossum from department of Loreto, Peru. The endoparasites were: Nematoda: Aspidodera sp., Cruzia tentaculata, Physaloptera mirandai, Physaloptera sp., Pterygodermatites sp., Trichuris sp., Turgida turgida, and Viannaia sp.; Trematoda: Podospathalium pedatum; Acanthocephala: Giganthorhynchus ortizi and Pentastomida: nymph. For the first time Trichuris sp., Pterygodematities sp., Turgida turgida, Viannaia sp. and Podospathalium pedatum are reported from marsupials in Peru. Also, for the first time the following parasite-hosts associations are recorded: Pterygodermatites sp.-Marmosa regina, Viannaia sp.-Marmosops noctivagus, Trichuris sp.-Marmosops cf. noctivagus, Podospathalium pedatum-Monodelphis adusta, Giganthorhynchus ortizi-Marmosops cf. noctivagus, and nymphs of pentastomida-Marmosa regina and Metachirus nudicaudatus.

Keywords: wildlife, parasites, marsupials, Amazon, Loreto, Peru.

\section{Introducción}

Perú es uno de los países con mayor diversidad de mamíferos; de las 508 especies nativas registradas 40 pertenecen al orden Didelphimorphia de las cuales 29 están presentes en la selva baja (Pacheco et al. 2009). La Amazonia en el noreste de Perú es una de las área con mayor diversidad y abundancia de marsupiales, con 13 especies colectadas sólo en la Estación Allpahuayo Mishana, Loreto (Hice 2003, Díaz y Willig 2004).

A pesar de los muestreos realizados durante muchos ańos, no existen registros de los endoparásitos presentes en marsupiales para el noreste de Perú. Los estudios de helmintos de marsupiales realizados en el Perú se han llevado a cabo principalmente en el noroeste (departamentos Cajamarca, San Martín, Loreto), en la región central (departamentos de Junín, Huánuco) y sureste (departamentos de Cusco y Madre de Dios). Hasta el momento se han registrado como huéspedes de helmintos a cuatro especies de marsupiales: Didelphis albiventris (Temminck 1825) donde se han registrado dos especies de endoparásitos, Didelphis marsupialis Linnaeus 1758 con 17 especies, Metachirus nudicaudatus (Desmarest 1817) con seis, Philander opossum (Linnaeus, 1758) con nueve y Monodelphis emiliae (Thomas 1912) con dos (Tantaleán \& Chávez 2004, Tantaleán et al. 2005). Esto confirma que los marsupiales en Perú poseen una gran variedad de formas parasitarias.

Los parásitos constituyen una fuente rica de información sobre la biología y otros aspectos importantes de sus huéspedes, ya que con la finalidad de permanecer en ellos han tenido que sortear grandes problemas. Es por ello que han desarrollado ciclos vitales complejos que les facilite, principalmente, ser ingeridos por sus huéspedes, de tal manera que su presencia nos permite conocer aspectos alimentarios del huésped o de la biota que los rodea, siendo su conocimiento de gran valor. Por otro lado, el conocimiento de los parásitos de animales de vida silvestre en el Perú es muy reducido; por este motivo, en este trabajo entregamos en una primera etapa y como base para futuros estudios, nuevas relaciones huésped-parásito, teniendo como huéspedes a marsupiales.

\section{Material y métodos}

Se colectaron un total de 370 marsupiales entre los meses de diciembre de 2002 y diciembre de 2005. Las localidades de colectadas fueron seleccionadas a lo largo de la carretera Iquitos-Nauta, departamento de Loreto; incluyendo diferentes tipos de ambientes: bosque primario y secundario y áreas rurales (zonas de cultivo, criaderos de chanchos, etc.). Las trampas fueron colocadas en transectas de $500 \mathrm{~m}$ en cada uno de los ambientes, con estaciones separadas cada $10 \mathrm{~m}$ con dos trampas (una Sherman y una Tomahawk) en cada estación; las trampas fueron cebadas con avena o frutas. También se usaron trampas de caídas, entre 5 a 9 baldes.

Los datos de medidas externas, sexo, condición reproductiva se obtuvieron de cada uno de los ejemplares capturados siguiendo a Díaz et al. (1998). Parte de los especímenes fueron depositados 
en el Museo de Historia Natural de la Universidad Nacional de San Marcos (UNMSM), Lima, Perú y parte en la Colección Mamíferos Lillo (CML), Tucumán, Argentina; el material aún se encuentra en proceso de catalogado por lo que los especimenes se designan con los números de campo del colector y sus iniciales MMD (M. Mónica Díaz).

Para la obtención de los endoparásitos se realizó una búsqueda directa en los órganos y además se extrajo el estómago e intestinos para una posteriormente disección. En total fueron examinados 40 individuos de 8 especies: 3 Caluromys lanatus (Olfers 1818), 1 Didelphis marsupialis, 9 Marmosops noctivagus (Tschudi 1844), 11 Metachirus nudicaudatus, 6 Marmosa (Micoureus) regina Thomas 1898, 1 Monodelphis adusta Thomas 1897, 3 Philander andersoni (Osgood 1913) y 6 Philander opossum. Los helmintos obtenidos se colocaron en formol 10\%, los nematodos, acantocéfalos y ninfas de pentastómidos se clarificaron en una mezcla de alcohol con fenol, y los tremátodes se colorearon con carmín de Semichon de acuerdo a la técnica convencional.

\section{Resultados}

A continuación, se describen para cada endoparásito colectado: a) huésped: especie hospedadora entre paréntesis iniciales y número del colector, sexo, fecha de colecta; b) localidad de colecta: localidad completa con coordenadas y tipo de ambiente de colecta; c) localización del parásito en el huésped y d) comentarios.

\section{Nematoda}

\section{Familia Aspidoderidae SkrJabin y Schikhobalova 1947 [FREITAS 1956]}

\section{Aspidodera sp.}

Huésped: Marmosops cf. noctivagus (MMD 4503, macho, 21 marzo 2005).

Localidad de colecta: Aproximadamente a $500 \mathrm{~m} \mathrm{E} \mathrm{km}$ 28,8 de la carretera Iquitos-Nauta, 3059,227'S-73²4,920 W (bosque secundario).

Localización: Intestino grueso.

Huésped: Metachirus nudicaudatus (MMD 4316, hembra, 22 febrero 2005).

Localidad de colecta: Ex Petroleros, $300 \mathrm{~m} \mathrm{~W} \mathrm{~km} \mathrm{39,8} \mathrm{de}$ la carretera Iquitos-Nauta, $4^{\circ} 04,740^{\prime}-S 73^{\circ} 27,180^{\prime} \mathrm{W}$ (bosque primario).

Localización: Intestino grueso.

Huésped: Metachirus nudicaudatus (MMD 4799, hembra, 7 junio 2005).

Localidad de colecta: $22,9 \mathrm{~km}$ E del $\mathrm{km} \mathrm{28,8}$ de la carretera Iquitos-Nauta (caserío Palo Seco) 3059,749'S-73²4,359'W (bosque secundario).

Localización: Intestino grueso. 2005).

Huésped: Marmosa regina: (MMD 4461, hembra, 16 marzo

Localidad de colecta: aproximadamente a $500 \mathrm{~m} \mathrm{E} \mathrm{km}$ 28,8 de la carretera Iquitos-Nauta, 3059,227'S-73²4,920'W (bosque primario).
Localización: Intestino grueso.

Huésped: Philander opossum (MMD 4291, hembra, 18 febrero 2005).

Localidad de colecta: Ex Petroleros, $300 \mathrm{~m} \mathrm{~W} \mathrm{~km} \mathrm{39,8} \mathrm{de}$ la carretera Iquitos-Nauta, $4^{\circ} 04,740^{\prime} \mathrm{S}-73^{\circ} 27,180^{\prime} \mathrm{W}$ (bosque secundario).

Localización: Intestino grueso.

Comentario: Este género parasita en roedores, edentados y marsupiales; posee numerosas especies muchas de las cuales fueron registradas en marsupiales como en Caluromys derbianus (citado como Philander laniger pallidus por Vicente 1966), Didelphis albiventris (citado como D. paraguayensis por Vicente 1966), D. aurita, D. marsupialis (citado como D. mesamericana por Vicente, 1966), D. virginiana, Marmosa murina, Metachirus nudicaudatus, Chironectes minimus (Vicente 1966, Díaz Hungría 1978, Navone \& Suriano 1992a, Quintão e Silva \& Martins de Araujo Costa 1999, Thatcher 2006, Chagas-Montinho 2007).

Varios trabajos se han realizado sobre las especies de Aspidodera que parasitan en marsupiales, así como revisiones del género, la última fue realizada por Santos et al. (1990) quienes resaltan la importancia de los cordones cefálicos en la identificación de las especies. En Perú, solo se conoce la presencia de $A$. harwoodi Chandler 1932 parasitando el ciego de D. marsupialis en el departamento de Huanuco. Debido a que el material estudiado se encontraba en mal estado no se pudieron identificar los especimenes a nivel de especie.

\section{Familia Kathklaniddae Travassos 1918}

\section{Cruzia tentaculata (Rudolphi 1819) Travassos 1917}

Huésped: Metachirus nudicaudatus: (MMD 4316, hembra, 22 febrero 2005).

Localidad de colecta: Ex Petroleros, $300 \mathrm{~m} \mathrm{~W} \mathrm{~km} \mathrm{39,8} \mathrm{de}$ la carretera Iquitos-Nauta, $4^{\circ} 04,740^{\prime} \mathrm{S}-73^{\circ} 27,180^{\prime} \mathrm{W}$ (bosque primario).

Localización: Intestino.

Comentario: Esta especie parasita a reptiles, anfibios y mamíferos como los marsupiales. En el Perú, previamente había sido reportada solo en D. marsupialis procedente de Cajamarca (Sarmiento et al. 1999), y de en un individuo en cautiverio (Arrojo 2002). En otros países fue registrada en D. marsupialis, $D$. albiventris, $D$. aurita, $D$. virginiana, $M$. nudicaudatus y $P$. opossum (Adnet et al. 2009, Quintão e Silva y Martins de Araujo Costa 1999, Gomes et al. 2003, Monet-Mendoza et al. 2005, Thatcher 2006).

\section{Familia Physalopteridae Railliet 1893 [Leiper 1908]}

\section{Physaloptera mirandai Lent y Freitas 1937}

Huésped: Metachirus nudicaudatus (MMD 4778 macho, 5 junio 2005; MMD 4799 hembra, 7 junio 2005).

Localidad de colecta: $22,9 \mathrm{~km}$ E del $\mathrm{km} \mathrm{28,8}$ de la carretera Iquitos-Nauta (caserío Palo Seco) 3059,749'S-73²4,359'W (bosque secundario).

Localización: Estómago. 
Comentario: Los especimenes estudiados poseian características concordantes con la descripción realizada por Lent y Freitas (1937) de especimenes colectados del estómago de Metachirus nudicaudatus personatus de Brasil.

\section{Physaloptera sp.}

Huésped: Metachirus nudicaudatus (MMD 3824 macho, 10 noviembre 2004).

Localidad de colecta: Paujil, W km 37,45 de la carretera Iquitos-Nauta $4^{\circ} 03,527^{\prime} \mathrm{S}-73^{\circ} 26,535^{\prime} \mathrm{W}$ (bosque secundario).

Localización: Boca del estómago.

Huésped: Metachirus nudicaudatus (MMD 4316, hembra, 22 febrero 2005).

Localidad de colecta: Ex Petroleros, $300 \mathrm{~m} \mathrm{~W} \mathrm{~km} \mathrm{39,8} \mathrm{de}$ la carretera Iquitos-Nauta, $4^{\circ} 04,740^{\prime} \mathrm{S}-73^{\circ} 27,180^{\prime} \mathrm{W}$ (bosque primario).

Localización: Estómago.

Huésped: Philander opossum (MMD 4479 hembra, 18 marzo 2005).

Localidad de colecta: Aproximadamente a $500 \mathrm{~m} \mathrm{E} \mathrm{km}$ 28,8 de la carretera Iquitos-Nauta, 3059,227'S-73024,920'W (bosque secundario).

\section{Localización: Estómago.}

Comentario: Especies de este nemátodo también fue encontrado parasitando a carnívoros Mustelidae y roedores Echimyidae (Sarmiento et al. 1999, Foster et al. 2007). Thatcher (2006) cita este género para $M$. nudicaudatus en Brasil y Castro-Arellano et al. (2000) en Philander de Paraguay.

\section{Familia Rictularildae Railliet 1916}

\section{Pterygodermatites sp.}

Huésped: Marmosa (Micoureus) regina (MMD 4461 hembra con crías, 16 marzo 2005).

Localidad de colecta: Aproximadamente a $500 \mathrm{~m} \mathrm{E} \mathrm{km}$ 28,8 de la carretera Iquitos-Nauta, 3059,227'S-73024,920'W (bosque primario).

Localización: Intestino delgado.

Comentario: Aunque por la falta de material en buen estado no se pudo identificar el subgénero y la especie, nuestros especimenes corresponden a un nemátodo poco frecuente en marsupiales. Ramallo y Claps (2007) mencionan a P. (Paucipectines) kozeki (Chabaud y Bain 1981) Navone 1989, en D. albiventris, Thylamys pusillus (Desmarest 1804) (citado como T. pusilla) y Lestodelphys halli (Thomas 1921) de Argentina, y Jiménez et al. (2008) lo mencionan en Thylamys pallidior (Thomas 1902) de Bolivia. En Argentina, también se ha señalado la presencia de $P$. (Paucipectines) spinicaudatis Navone y Suriano $1992 \mathrm{~b}$ en Dromiciops gliroides Thomas 1894 (citado como D. australis); del mismo modo, P. (Paucipectines) jägerskiöldi Lent y Freitas 1935 es la subespecie presente en marsupiales de Brasil (Lopes Torres et al. 2007). Hasta el momento este parásito no había sido registrado en $M$. (Micoureus) regina.

\section{Familia Trichuridae (RANSom 1911) RaILliet 1915}

\section{Trichuris sp.}

Huésped: Marmosops cf. noctivagus: (MMD 4503, macho, 21 marzo 2005).

Localidad de colecta: Aproximadamente a $500 \mathrm{~m} \mathrm{E} \mathrm{km}$ 28,8 de la carretera Iquitos-Nauta, 3059,227'S-73²4,920'W (bosque secundario).

Localización: Intestino grueso.

Huésped: Philander andersoni (MMD 4803, macho, 10 junio 2005).

Localidad de colecta: $2,9 \mathrm{~km}$ E del $\mathrm{km} \mathrm{28,8}$ de la carretera Iquitos-Nauta (caserío Palo Seco) 3059,749'S-73²4,359'W (bosque secundario)

Localización: Intestino grueso.

Huésped: Philander opossum (MMD 4291, hembra, 18 febrero 2005).

Localidad de colecta: Ex Petroleros, $300 \mathrm{~m} \mathrm{~W} \mathrm{~km} \mathrm{39,8} \mathrm{de}$ la carretera Iquitos-Nauta, $4^{\circ} 04.740^{\prime} \mathrm{S}-73^{\circ} 27,180^{\prime} \mathrm{W}$ (bosque secundario).

Localización: Intestino grueso.

Comentario: Solo se encontraron ejemplares hembras por lo que no se pudo identificar a nivel de especie. Este género fue registrado en D. albiventris en Brasil (Quintão e Silva y Martins de Araujo Costa 1999) y en P. opossum (Castro-Arellano et al. 2000) en varios países pero no en el Perú. No se encontraron referencias de la presencia de este parásito en el género Marmosops.

\section{Turgida turgida (Rudolphi 1819) Travassos 1920}

Huésped: Philander opossum (MMD 3769 hembra con crías, 28 octubre 2004).

Localidad de colecta: San Lucas, W km 43 de la carretera Iquitos-Nauta, $4^{\circ} 06,247^{\prime} \mathrm{S}-73^{\circ} 27,791^{\prime} \mathrm{W}$ (bosque secundario).

Localización: Estómago.

Comentario: Esta especie es ampliamente conocida y parasita el estómago produciendo grandes úlceras, localizándose principalmente en la curvatura mayor (Gray \& Anderson 1982); sus caracteres taxonómicos han sido establecidos por Travassos (1920) y estudiados en detalle en microscopia de barrido (Matey et al. 2001). Este registro es el primero de la especie para el Perú. En otros países T. turgida ha sido registrada en $D$. albiventris, $D$. virginiana, $D$. aurita, D. marsupialis, $P$. opossum y M. nudicaudatus (Travassos 1932, Díaz Hungría 1978, Gray y Anderson 1982, Quintão e Silva y Martins de Araujo Costa 1999, Gomes et al. 2003)

\section{Familia Viannaildae Durette-Desset y Chabaud 1981}

\section{Viannaia sp.}

Huésped: Marmosops noctivagus (MMD 4451, hembra, 16 marzo 2005).

Localidad de colecta: Aproximadamente a $500 \mathrm{~m} \mathrm{E} \mathrm{km}$ 
28,8 de la carretera Iquitos-Nauta, 3059,227'S-7324,920'W (bosque secundario).

Localización: Intestino delgado.

Huésped: Metachirus nudicaudatus: (MMD 4799, hembra, 7 junio 2005)

Localidad de colecta: 2,9 km E del km 28,8 de la carretera Iquitos-Nauta (caserío Palo Seco) 3059,749'S-73²4,359'W (bosque secundario).

Localización: Intestino grueso.

Comentario: Solo se encontraron tres especimenes hembras en los ejemplares examinados. Este género fue registrado en $D$. aurita, D. albiventris, D. virginiana, $M$. nudicaudatus y $P$. opossum en Brasil (Quintão e Silva y Martins de Araujo Costa 1999, Gomes et al. 2003, Monet-Mendoza et al. 2005, Thatcher 2006), pero aparentemente, no se conocen registros de este nemátodo en el género Marmosops.

\section{TREMATODA}

\section{Familia Diplostomidae Poirier, 1886}

\section{Podospathalium pedatum (Diesing, 1850) Dubois, 1932}

Huésped: Monodelphis adusta (MMD 4305, macho, 20 febrero 2005).

Localidad de colecta: Ex Petroleros, $300 \mathrm{~m} \mathrm{~W} \mathrm{~km} \mathrm{39,8} \mathrm{de}$ la carretera Iquitos-Nauta, $4^{\circ} 04,740^{\prime} \mathrm{S}-73^{\circ} 27,180^{\prime} \mathrm{W}$ (bosque primario).

Localización: Intestino.

Huésped: Philander opossum (MMD 3525, macho, 10 julio 2004).

Localidad de colecta: Camino a El Paujil, 1,8 km al W del $\mathrm{km} 35$ de la carretera Iquitos-Nauta, $4^{\circ} 01,217^{\prime} \mathrm{S}-73^{\circ} 26,787^{\prime} \mathrm{W}$ (bosque primario).

Localización: Intestino.

Huésped: Philander opossum (MMD 3528, hembra, 10 julio 2004).

Localidad de colecta: Camino a El Paujil, $1,8 \mathrm{~km}$ al W del $\mathrm{km} 35$ de la carretera Iquitos-Nauta, $4^{\circ} 01,217^{\prime} \mathrm{S}-73^{\circ} 26,787^{\prime} \mathrm{W}$ (bosque secundario).

Localización: Intestino.

Comentario: Parásito intestinal; en América del Sur solo se ha registrado en Didelphis, Metachirus y Philander (Collins 1973, Thatcher 2006), por lo que Monodelphis adusta es un nuevo huésped.

\section{ACANTHOCEPHALA}

\section{Clase Archiacanthocephala Meyer 1931}

Familia Gigantorhynchidae Hamann 1892

\section{Gigantorhynchus ortizi Sarmiento 1954}

Huésped: Marmosops cf. noctivagus (MMD 4503, macho, 21 marzo 2005).
Localidad de colecta: Aproximadamente a $500 \mathrm{~m} \mathrm{E} \mathrm{km}$ 28,8 de la carretera Iquitos-Nauta, 359,227'S-73²4,920'W (bosque secundario).

Localización: Intestino.

Huésped: Metachirus nudicaudatus (MMD 3502, macho, 6 julio 2004).

Localidad de colecta: Camino a El Paujil, $1,8 \mathrm{~km}$ al W del $\mathrm{km} 35$ de la carretera Iquitos-Nauta, $4^{\circ} 01,217^{\prime} \mathrm{S}-73^{\circ} 26,787^{\prime} \mathrm{W}$ (bosque primario)

Localización: Intestino (Fig. 1).
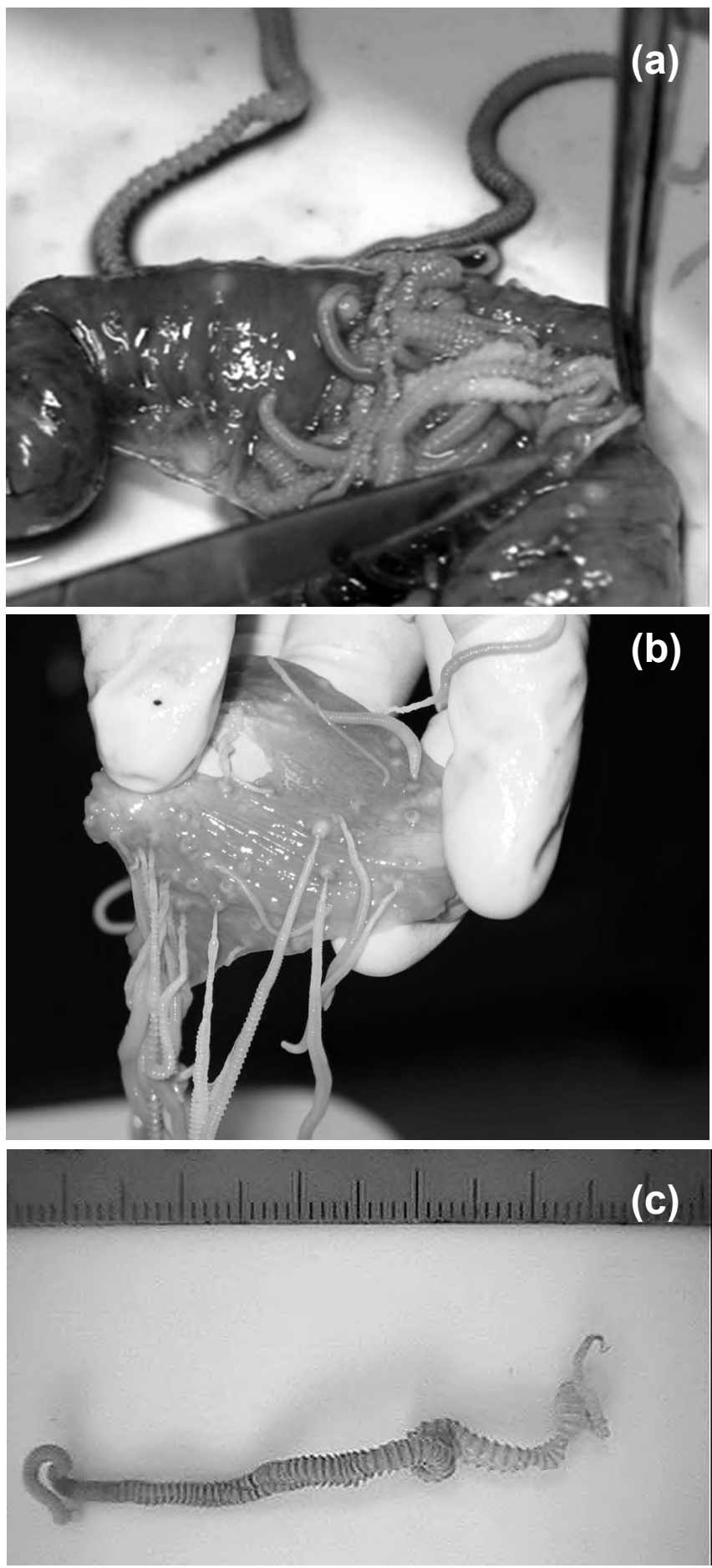

Figura 1. Gigantorhynchus ortizi (a) en el intestino, (b) unido a la pared interna del intestino, y (c) ejemplar aislado, todos del intestino de Metachirus nudicaudatus (MMD 3502). 
Tabla 1.Se indica parásito, hospedador, localización del parásito en el hospedador y ambiente donde fue colectado el huésped (BP: bosque primario, BS: bosque secundario). * Primer registro en esta especie como huésped del parásito.

\begin{tabular}{|c|c|c|c|}
\hline Parásito & Hospedador & Localización & Ambiente \\
\hline \multirow{4}{*}{ Aspidodera sp. } & Marmosops cf. noctivagus & \multirow{4}{*}{ Intestino grueso } & BS \\
\hline & Metachirus nudicaudatus & & $\begin{array}{l}\text { BS } \\
\text { BP }\end{array}$ \\
\hline & Marmosa regina & & $\begin{array}{l}\mathrm{BP} \\
\mathrm{BP}\end{array}$ \\
\hline & Philander opossum & & BS \\
\hline Cruzia tentaculata & Metachirus nudicaudatus & Intestino & BP \\
\hline Physaloptera mirandai & Metachirus nudicaudatus & Estómago & BS \\
\hline \multirow{3}{*}{ Physaloptera sp. } & \multirow{2}{*}{ Metachirus nudicaudatus } & \multirow{2}{*}{ Boca del estómago, Estómago } & BS \\
\hline & & & BP \\
\hline & Philander opossum & Estómago & BS \\
\hline Pterygodermatites sp. & Marmosa regina* & Intestino & $\mathrm{BP}$ \\
\hline \multirow{3}{*}{ Trichuris sp. } & Marmosops cf. noctivagus & \multirow{3}{*}{ Intestino grueso } & \multirow{3}{*}{ BS } \\
\hline & Philander andersoni & & \\
\hline & Philander opossum & & \\
\hline Turgida turgida & Philander opossum & Estómago & BS \\
\hline \multirow{2}{*}{ Viannaia sp. } & Marmosops noctivagus* & Intestino delgado & \multirow{2}{*}{ BS } \\
\hline & Metachirus nudicaudatus & Intestino grueso & \\
\hline \multirow{3}{*}{ Podospathalium pedatum } & Monodelphis adusta* & \multirow{3}{*}{ Intestino } & $\mathrm{BP}$ \\
\hline & & & $\mathrm{BP}$ \\
\hline & Philander opossum & & BS \\
\hline \multirow{2}{*}{ Giganthorhynchus ortizi } & Marmosops cf. noctivagus* & \multirow{2}{*}{ Intestino } & BS \\
\hline & Metachirus nudicaudatus & & $\mathrm{BP}$ \\
\hline \multirow{5}{*}{ Pentastomida (ninfas) } & \multirow{3}{*}{ Metachirus nudicaudatus* } & Sin datos & $\mathrm{BP}$ \\
\hline & & Pulmones, hígado y pared del estómago & \\
\hline & & Pared del estómago & BS \\
\hline & Marmosa regina* & Estómago & $\mathrm{BP}$ \\
\hline & Philander opossum & Pared del útero & BS \\
\hline
\end{tabular}

Comentario: Este acantocéfalo se ha encontrado previamente en Perú en $M$. nudicaudatus proveniente de La Merced, departamento de Junin; pero otra especie del mismo género, G. lutzi Machado, 1941 fue registrada para D. marsupialis en Tingo María, departamento de Huanuco (Tantaleán et al. 2005). De acuerdo a nuestro conocimiento, hasta la fecha no se ha reportado a Marmosops como huésped de alguna especie de Gigantorhynchus.

\section{Pentastomida (ninfa)}

Huésped: Metachirus nudicaudatus (MMD 785, hembra, 20 enero 2003).

Localidad de colecta: Moralillo, $1.5 \mathrm{~km}$ E $500 \mathrm{~m} \mathrm{~S}$ del km 15,2 de la carretera Iquitos-Nauta, 3054,379'S-73020,617'W (bosque primario).

Localización: Sin datos.

Huésped: Metachirus nudicaudatus (MMD 3782, hembra, 30 octubre, 2004).

Localidad de colecta: San Lucas, W km 43 de la carretera Iquitos-Nauta, $4^{\circ} 06,247^{\prime} \mathrm{S}-73^{\circ} 27,791^{\prime} \mathrm{W}$ (bosque secundario).

Localización: Pulmones, hígado y pared del estómago.

Huésped: Metachirus nudicaudatus (MMD 3821, hembra, 10 noviembre 2004).

Localidad de colecta: Paujil, W km 37,45 de la carretera Iquitos-Nauta, $4^{\circ} 03,527^{\prime} \mathrm{S}-73^{\circ} 26,535^{\prime} \mathrm{W}$ (bosque secundario).

Localización: Pared del estómago.
Huésped: Marmosa (Micoureus) regina (MMD 4461, hembra, 16 marzo 2005).

Localidad de colecta: Aproximadamente a $500 \mathrm{~m} \mathrm{E} \mathrm{km}$ 28,8 de la carretera Iquitos-Nauta, 3059,227'S-73²4,920'W (bosque primario).

Localización: Estómago.

Huésped: Philander opossum (MMD 3792, hembra, 31 octubre 2004).

Localidad de colecta: San Lucas, W km 43 de la carretera Iquitos-Nauta, $4^{\circ} 06,247^{\prime} \mathrm{S}-73^{\circ} 27,791^{\prime} \mathrm{W}$ (bosque secundario).

Localización: Pared del útero.

Comentario: Los estadios adultos de pentastómidos son principalmente parásitos de serpientes, las ninfas se encuentran en órganos parenquimatosos de diversos mamíferos. En el Perú, hasta el momento no se había reportando presencia de estas formas en marsupiales. Solo existe un registro de infección por larvas de pentastómido en Philander opossum (Castro-Arellano et al. 2000), por lo que $M$. (Micoureus) regina y $M$. nudicaudatus serían nuevos huéspedes.

\section{Discusión}

Los marsupiales infectados están representados por cinco géneros y seis especies, colectados en bosque secundario o primario (Tabla 1). Tres especimenes estaban parasitados por tres diferentes especies de parásitos y dos por dos especies. Metachirus nudicaudatus (MMD 4316) infectado con Aspidodera sp., Cruzia sp. y Physaloptera sp.; otro ejemplar de la misma especie (MMD 
4799) por Aspidodera sp., Physaloptera mirandai y Viannaia sp. y un Marmosops cf. noctivagus (MMD 4503) por Aspidoptera sp., Gygantorhynchus ortizi y Trichuris sp. Mientras que en el espécimen de Philander opossum (MMD 4291) se encontraron Aspidoptera sp. y Trichuris sp.; y en M. (Micoureus) regina (MMD 4461) Aspidoptera sp. y ninfas de pentastomidos.

De los huéspedes infectados, los que tuvieron mayor número de individuos con parásitos fueron $M$. nudicaudatus con ocho animales infectados y $P$. opossum con seis. La segunda fue la especie más común en el área de estudio con un total de 153 ejemplares colectados contra solo 33 de $M$. nudicaudatus; por lo que se podría concluir que el grado de infección (25\%) en $M$. nudicaudatus es importante para el área de estudio.

Los siguientes helmintos son nuevos registros para marsupiales del Perú: Trichuris sp., Pterygodematities sp., Turgida turgida, Viannaia sp. y Podospathalium pedatum; asimismo, las subsiguientes asociaciones parásitos-huéspedes se citan por primera vez: Pterygodermatites sp.-Marmosa (Micoureus) regina, Viannaia sp.-Marmosops noctivagus, Trichuris sp.-Marmosops cf. noctivagus, Podospathalium pedatum-Monodelphis adusta, Giganthorhynchus ortizi-Marmosops cf. noctivagus, y ninfas de pentastómidosMarmosa (Micoureus) regina y Metachirus nudicaudatus.

\section{Agradecimientos}

Agradecemos a Victor Linares, Sixto Mananita, Cesar Ahuanari y Rubi Angulo estudiantes de la Universidad Nacional de San Marcos, por su invalorable ayuda tanto en el trabajo de campo como de laboratorio. El trabajo de campo fue realizado gracias al subsidio de Public Health Service National Institute of Allergy and Infectious Diseases, Estados Unidos (subsidios R01TW005860, D43TW007120 y K24AI068903). Un agradecimiento especial a Joseph Vinetz, el investigador principal del subsidio. También al Instituto Nacional de Recursos Naturales (INRENA), Ministerio de Agricultura de Perú por otorgar los permisos de colecta y captura de los ejemplares.

\section{Literatura citada}

Adnet F.A.O., D.H.S. Anjos, A. Menezes Oliveira \& R.M. Lanfredi. 2009. Further description of Cruzia tentaculata (Rudolphi, 1819) Travassos, 1917 (Nematoda: Cruzidae) by light and scanning electron microscopy. Parasitology Research 104: 1207-1211

Arrojo L. 2002. Parásitos de animales silvestres en cautiverio en Lima, Perú. Revista Peruana de Biología 9: 118-120.

Castro-Arellano I., H. Zarza \& R.A. Medellín. 2000. Philander opossum. Mammalian Species 638: 1-8.

Chagas-Moutinho V.A., A. Oliveira-Menezes, M.Q. Cárdenas \& R.M. Lanfredi. 2007. Further description of Aspidodera raillieti (Nematoda: Aspidoderidae) from Didelphis marsupialis (Mammalia: Didelphidae) by light and scanning electron microscopy Parasitology Research 101: 1331-1336.

Collins L.R. 1973. Monotremes and marsupials. A reference for zoological institutions. Smithsonian Institution Press. Washington, D.C.

Díaz M.M., D.A. Flores \& R.M. Barquez. 1998. Instrucciones para la preparación y conservación de mamíferos. PIDBA (Programa de Investigaciones de Biodiversidad Argentina), Publicaciones Especiales 1: 44 pp.

Díaz M.M. \& M.R. Willig. 2004. Nuevos registros de Glironia venusta y Didelphis albiventris (Didelphimorpia) para Perú. Mastozoología Neotropical 11: 185-192.
Díaz Hungría C. 1978. Helmintos parásitos de vertebrados en el estado Zulia (Venezuela) algunas especies nuevas para Venezuela. Veterinaria Tropical 3:15 - 37.

Foster G.W., M.W. Cunningham, J.M. Kinsella \& M. Owen. 2007. Parasitic helminthes of free-ranging mink (Neovison vison mink) from Southern Florida. Journal of Parasitology 93: 945-946.

Gomes D.C., R.P. da Cruz, J.J. Vicente \& R.M. Pinto. 2003. Nematode parasites of marsupials and small rodents from the Brazilian Atlantic Forest in the State of Rio de Janeiro, Brazil. Revista Brasileria de Zoologia 20(4): 699-707 .

Gray J.B. \& R.C. Anderson. 1982. Observations on Turgida turgida (Rudolphi, 1819) (Nematoda: Physalopteroidea) in the american opossum (Didelphis virginiana). Journal of Wildlife Diseases 18: 279-285.

Hice C. L. 2003. The non-volant mammals on the Estación Biológica Allpahuayo assessment of the natural history and community ecology of a proposed reserve. Doctoral Dissertation Thesis. Faculty of Texas Tech University $304 \mathrm{pp}$.

Jiménez F.A., J.K. Braun, M.L. Campbell \& S.L. Gardner. 2008. Endoparasites of fat-tailed mouse opossums (Thylamys: Didelphidae)from northwestern Argentina and southern Bolivia, with the description of a new species of tapeworm. J. Parasitol.94(5): 1098-1102

Lent H. \& J.Freitas. 1937. Nova Physaloptera parasite de marsupial. Nematoda: Spiruroidea. Memorias do Instituto Oswaldo Cruz 32: 221-223.

Lopes Torres E., A. Maldonado \& R.M. Lanfredi. 2007. Pterygodermatites (Paucipectines) jägerskiöldi (Nematoda: Rictulariidae) from Gracilinanus agilis and G. microtarsus (Marsupialia: Didelphidae) in Brazilian pantanal and atlantic forest by light and scanning electron microscopy. Journal of Parasitology 93: 274-279.

Matey V.E., B.I. Cuperman \& J.M. Kinsella. 2001. Scanning electron microscopy of Turgida turgida (Nematoda: Spiruroidea), parasite of the Virginia opossum, Didelphis virginiana, from southern California. Journal of Parasitology 87: 1199-1202.

Monet-Mendoza A., D. Osorio-Sarabia \& L. García-Prieto. 2005. Helminths of the Virginia Opossum Didelphis virginiana (Mammalia: Didelphidae) in Mexico. Journal of Parasitology 91: 213-219.

Navone G. T. \& D. M. Suriano. 1992a. Composición de especies y dinámica estacional de la comunidad de helmintos que parasita a Didelphis albiventris (Marsupiala: Didelphidae) en las sabanas del centro de Argentina. Ecología Austral 2: $95-100$

Navone G.T. \& D.M. Suriano. 1992b. Perygodermatites (Paucipectines) spinicaudatis n. sp. (Nematoda: Rictularidae) from Dromiciops australis (Marsupialia: Microbiotheriidae) in Bariloche, Rio Negro, Argentina. Biogeographical distribution and host-parasites relationships. Memorias do Instituto Oswaldo Cruz 87: 533-538.

Pacheco V., R. Cadenillas, E. Salas, et al. 2009. Diversidad y endemismo de los mamíferos del Perú. Rev. peru. biol. 16(1): 005-032.

Quintão e Silva M.G. \& H.M. de Araujo Costa. 1982. Helminths of White-bellied opossum from Brazil. Journal of Wildlife Diseases 35(2): 371-374.

Ramallo G. \& L.E. Claps. 2007. Nuevos hospedadores y registros geográficos de Pterygodermatites (Paucipectines) kozeki (Nematoda (Rictularidae) en Argentina. Mastozoología Neotropical 14: 93-96.

Santos C.P., H. Lent \& D.C. Gomes. 1990. The genus Aspidodera Railliet and Henry, 1912 (Nematoda: Heterakoidea): Revision, new genus and key for species. Revista Brasileira de Biología, Rio de Janeiro 50: 1017-1031. 
Sarmiento L., M. Tantaleán \& A. Huiza. 1999. Nemátodos parásitos del hombre y de los animales en el Perú. Revista Peruana de Parasitología 14: 9-65.

Tantaleán M., L. Sánchez, L. Gómez \& A. Huiza. 2005. Acantocéfalos del Perú. Revista Peruana de Biología 12: 83-92.

Tantaleán M. \& J. Chavez 2004. Wild animals endoparasites (Nemathelminthes and Plathelminthes) from the Manu Biosphere Reserve, Peru. Revista Peruana de Biología 11: 219-222.
Thatcher V.E. 2006. Os Endoparasitos dos Marsupiais Brasileiros. En: Cáceres N.C. y E.L.A. Monteiro-Filho, eds. Os Marsupiais do Brasil: Biologia, Ecologia e Evolução, Editora UFMS, Campo Grande. PP. 53-68.

Travassos L. 1920. Contribuções para o conhecimento da fauna helmintológica brasileira. X. Sobre as espécies do género Turgida. Memorias do Instituto Oswaldo Cruz 12: 73-77.

Vicente J.J. 1966. Revisão da subfamilia Aspidoderinae Skjabin \& Shikhobalova, 1947 (Nematoda). Memórias do Instituto Oswaldo Cruz, 64 (fascículo único) 131-161. 


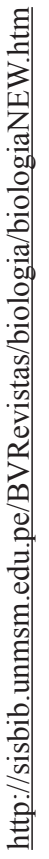

